\title{
EFEITO DA TOPOGRAFIA E DA PRECIPITAÇÃO NA FLORÍSTICA E NA PRODUÇÃO DE LITEIRA EM CAXIUANÃ, PARÁ ${ }^{1}$
}

\author{
Laura Suellen Ferreira², José Henrique Cattânio ${ }^{3}$ e Mário Augusto G. Jardim ${ }^{4}$
}

\begin{abstract}
RESUMO - O objetivo deste trabalho foi avaliar o efeito da topografia e da precipitação pluviométrica na composição arbórea e na produção de liteira em uma floresta ombrófila densa na Floresta Nacional de Caxiuanã. Foram demarcadas três parcelas de $1.000 \mathrm{~m}^{2} \mathrm{em}$ cada nível topográfico, caracterizado como baixio, intermediário e platô, bem como identificados os indivíduos arbóreos e coletadas amostras da liteira. Nos três níveis, foram registradas 124 espécies em 33 famílias, sendo estas Sapotaceae, Lecythidaceae e Chrysobalanaceae, que apresentaram o maior índice de valor de importância. Lecythis idatimon Aubl., Rinorea guianensis Aubl. e Eschweilera coriacea (DC.) S.A. Mori. A sazonalidade interferiu expressivamente na produção da liteira, revelando a maior produção no final da estação chuvosa e no início da estação seca. O estímulo ambiental para a queda das folhas é, principalmente, devido à diminuição da umidade relativa do ar, justificada pela necessidade das plantas em aumentar a eficiência fotossintética. A diferença na estrutura da população revela estratégias distintas para a produção de flores e, consequentemente, na dispersão de frutos e sementes.
\end{abstract}

Palavras-chave: Variação topográfica; Sazonalidade; Produção de liteira.

\section{THE EFFECT OF TOPOGRAPHY AND RAINFALL IN FLORISTIC AND LITTER PRODUCTION IN THECAXIUANÃ, PARÁ}

\begin{abstract}
The objective of this work was to study the effect of topography and rainfall in the structure of trees and litter production in a tropical rain forest in the National Forest of Caxiuanã. Were delimited three tranches of $1.000 \mathrm{~m}^{2}$ in each topographic levels (lowland, intermediate and plateau) and identified all individuals and collection of litter. For the three levels was recorded a total of 124 species belonging to 33 botanical families. The three families with higher importance value index for topographies of lowland and intermediate were Sapotaceae, Lecythidaceae and Chrysobalanaceae and for plateau were Lecythidaceae, Sapotaceae and Chrysobalanaceae. The species Lecythis idatimon Aubl., Rinoria guianensis Aubl. e Eschweilera coriacea (DC.) S.A. Mori. showed the greatest importance value indices for the three topographies. The seasonality affects significantly the litter production, showing the highest values at the end of rainy season and early dry season. The environmental stimulus for the fall of the leaves is mainly due to decrease in relative humidity, justified by the need of the plants to increase photosynthetic efficiency. The difference in population structure show strategies for flower production, and consequently the dispersion of fruits and seeds.
\end{abstract}

Keywords: Topographic variation; Seasonality; Litter production.

\footnotetext{
${ }^{1}$ Recebido em 13.01.2015 aceito para publicação em 29.10.2015.

${ }^{2}$ Ministério da Ciência e Tecnologia, Museu Paraense Emilio Goeldi, Parque Zoobotânico, Belém, PA - Brasil. E-mail: $<$ lauraferreira@museu-goeldi.br>.

${ }^{3}$ Universidade Federal do Pará, Faculdade de Meteorologia, Campus Universitário do Guamá, Belém, PA - Brasil. E-mail: $<$ cattanio@ufpa.br>.

${ }^{4}$ Ministério da Ciência e Tecnologia, Museu Paraense Emílio Goeldi, Coordenação de Botânica, Belém, PA - Brasil. E-mail: $<$ jardim@museu-goeldi.br>.
} 


\section{INTRODUÇÃO}

A liteira abrange o material depositado sobre o solo ainda não totalmente decomposto, que inclui principalmente folhas, flores, frutos, cascas e, em menor proporção, os resíduos de animais (DIAS; OLIVEIRAFILHO, 1997). A produção de liteira representa parte importante do total de produção primária nas florestas tropicais, sendo indispensável mecanismo de transferência de nutrientes entre a vegetação e o solo para a sustentabilidade das florestas, principalmente daquelas que crescem em regiões tropicais, cujo solo é pobre em nutrientes (VITOUSEK; SANFORD, 1986; NOVAIS; BARROS, 1997).

A quantidade de liteira depositada pode variar dentro de um mesmo tipo de vegetação. Segundo Werneck et al. (2001), a maioria dos ecossistemas florestais apresenta produção contínua de liteira durante todo o ano, cuja quantidade total produzida nas diferentes épocas depende do tipo de vegetação. A variação estacional da deposição de liteira é resultante da interação entre a vegetação e o clima. Vários fatores abióticos influenciam a produção de liteira como a energia radiativa, altitude, temperatura do ar, precipitação, disponibilidade hídrica, estoque de nutrientes no solo, umidade do solo e vento (BURGHOUTS et al., 1994; PORTES et al., 1996; DIAS; OLIVEIRA-FILHO, 1997).

A precipitação, a temperatura, o fotoperíodo, a intensidade de radiação, a qualidade do solo e a presença ou ausência de animais e predadores de flores e frutos, bem como os dispersores de sementes, estão diretamente relacionados com queda foliar, floração e frutificação das plantas (PEDRONI et al., 2002). Nas florestas tropicais, a fenologia pode ser menos sensível à temperatura e mais sintonizada com as mudanças sazonais da precipitação (REICH, 1995; SANCHEZ-AZOFEIFA et al., 2003), podendo variar de região para região(CUBASCH et al., 2001). Para áreas onde padrões de precipitação são fortemente influenciados pelo El Niño, como a Amazônia, as mudanças no clima podem afetar a frequência e a intensidade dos eventos fenológicos e a produção de liteira nas florestas (ASNER et al., 2000). Os estudos realizados na região de Caxiuanã evidenciaram forte relação entre a dinâmica da biomassa vegetal e as variáveis micrometeorológicas (METCALFE et al., 2008; ARAGÃO et al., 2009; SILVA et al., 2009; CHAVE et al., 2010). Este estudo teve como objetivo avaliar o efeito da topografia e da sazonalidade na

Revista Árvore, Viçosa-MG, v.39, n.6, p.995-1005, 2015 produção de liteira em uma floresta ombrófila densa na Estação Científica Ferreira Penna, Município de Melgaço, Pará.

\section{MATERIAL E MÉTODOS}

O estudo foi desenvolvido na área do Programa de Pesquisa em Biodiversidade na Amazônia Oriental - PPBio, na Floresta Nacional de Caxiuanã, no Município de Melgaço (Pará-Brasil), sob as coordenadas 01 57'36" $\mathrm{S} \mathrm{e} 51^{\circ} 36^{\prime} 55^{\prime \prime} \mathrm{W}$. A topografia regional é plana, ligeiramente ondulada, com elevações de terra que variam de 19,0 a 47,0 $\mathrm{m}$ acima do nível do mar. O solo é Latossolo Amarelo de origem terciária, oligotrófico, argiloarenoso ácido e profundo (ALMEIDA et al., 1993). Segundo a classificação climática de Köppen-Geiger, o clima da região é do tipo tropical quente e úmido, considerado como subtipo climático “Am” (PEEL et al., 2007). Possui distribuição regular das chuvas durante o ano com estação chuvosa, que abrange os meses de janeiro, fevereiro e março, e a estação menos chuvosa, de setembro, outubro e novembro. A temperatura média é de aproximadamente $26^{\circ} \mathrm{C}$, com mínima e máxima variando de $22^{\circ} \mathrm{Ca} 32{ }^{\circ} \mathrm{C}$, respectivamente, e com umidade relativa média anual de cerca de $80 \%$ (COSTA et al., 2009).

Para a análise da composição florística, foram estabelecidas três parcelas de 10 x 100 m em cada nível topográfico, discriminados aqui como Baixio, Intermediário e Platô, segundo a metodologia de Almeida et al. (1993), e identificados, com o auxílio de um parabotânico, todos os indivíduos arbóreos, bem como mensurados o diâmetro da altura do peito (DAP) e" $10 \mathrm{~cm}$ e a altura total. Foi calculado o índice de Importância Relativa (IVI), segundo Matteucci et al. (1982), e analisadas apenas as espécies com IVIe" 4,0\%. No interior de cada parcela, foram distribuídos por sorteio 12 coletores, construídos com tubos de PVC e malha de sombrite, com área de $0,25 \mathrm{~m}^{2}$ cada, totalizando 108 coletores. As coletas foram realizadas mensalmente, no período de agosto de 2009 a julho de 2010. O material coletado foi colocado em sacos de papel, identificado com número, local e data de coleta e previamente seco em estufa a gás, no campo, para posterior transporte.

No laboratório, o material foi separado nas frações: a) foliar: constituindo de folhas e estípulas; b) galhos; c) flores: botões florais e flores; d) frutos: jovens e maduros; e e) miscelânea: material que não se pode identificar como proveniente de uma das frações 
anteriores. Depois de separado, o material foi seco em estufa a $65^{\circ} \mathrm{C}$ até atingir peso constante. A pesagem do material foi feita separadamente em balança analítica com precisão de $0,001 \mathrm{~g}$. Os dados de chuva foram coletados automaticamente em uma torre micrometeorológica em Caxiuanã. Foi aplicado o teste de normalidade, para verificar a distribuição dos dados mensais. Em seguida, procedeu-se à análise de covariância, a fim de verificar a relação na produção mensal de liteira (variável dependente) em relação à topografia (fator), levando em consideração o efeito da covariada precipitação mensal e temperatura mensal média, máxima e mínima, em nível de 5\% de significância.

\section{RESULTADOS}

\subsection{Composição Florística}

Sapotaceae, Lecythidaceae e Chrysobalanaceae obtiveram o maior índice de valor de importância nos três níveis topográficos. No baixio, foram registrados 138 indivíduos em 21 famílias, 38 gêneros e 59 espécies. As espécies com IVIe" 4,0\% foram Lecythis idatimon Aubl. (7,58\%), Vouacapoua americana Aubl. (4,92\%), Pouteria cladantha Sandwith (4,23\%) e Rinorea guianensis Aubl. (4,22\%). No intermediário, 151 indivíduos em 24 famílias, 47 gêneros e 73 espécies. As espécies com maior IVI foram Rinorea guianensis Aubl. (6,17\%), Eschweilera coriacea $(6,10 \%)$, Lecythis idatimon $(4,85 \%)$, Pouteria anomala $(4,52 \%)$ e Vouacapoua americana (4,02\%). No platô, 121 indivíduos em 61 espécies, 43 gêneros e 23 famílias. As espécies com maior IVI foram Vouacapoua americana (5,91\%), Couratari stellata A.C.Sm. (5,80\%), Eschweilera coriacea $(5,52 \%)$ e Lecythis idatimon $(5,45 \%)$ (Tabela 1$)$. A composição florística na topografia intermediária apresentou os maiores valores em número de indivíduos, espécies, gêneros e famílias. Em termos de espécie mais dominante, as três topografias se diferem entre si, com destaque para a Lecythis idatimon, a qual está entre as três espécies mais dominantes nos níveis topográficos. O índice de valor de importância também foi diferenciado nas três topografias, ficando a topografia intermediária com espécies exclusivas em relação às outras.

\subsection{Produção de Liteira}

O total da produção de liteira foi de 9,$44 ; 8,64$; $9,11 \mathrm{Mg} \mathrm{ha}^{-1}$ ano $^{-1}$ nos níveis de baixio, intermediário e platô, com variação média mensal de 785,0 23,9 ;
$719,4 \pm 20,5 ;$ e $760,8 \pm 23,1 \mathrm{~kg} \mathrm{ha}^{-1}$. Não houve variação significativa entre os níveis topográficos $(p>0,05)$. Entretanto, foi constatada intensa produção de liteira ( $\mathrm{p}<0,01)$ no mês de agosto, no baixio e platô $(1.327,4$ $\left.\pm 94,3 ; 1.326,5 \pm 98,4 \mathrm{~kg} \mathrm{ha}^{-1}\right)$ e no mês de setembro $\left(1.101,6 \pm 121,9 \mathrm{~kg} \mathrm{ha}^{-1}\right)$ no nível intermediário, mostrando diferença na produção de liteira durante os meses, em todos os níveis $\left(\mathrm{F}_{\text {baixio }}=26,2, \mathrm{p}<0,001 ; \mathrm{F}_{\text {intermediário }}=\right.$ $\left.28,3, \mathrm{p}<0,001 ; \mathrm{F}_{\text {Platô }}=26,9, \mathrm{p}<0,001\right)$. Observou-se que o aumento da produção de liteira iniciou em abril, coincidente com o maior pico de chuva (mais de 500 $\mathrm{mm}$ ) até agosto-setembro, nos primeiros meses da estação seca, quando a maior produção ocorreu ao final de estação chuvosa e início da estação seca (Figura 1). A menor produção foi constatada no meio da estação chuvosa, em todos os níveis topográficos. Com base na análise de covariância, o fator que influenciou a produção foi a precipitação $(\mathrm{p}<0,03)$, principalmente entre as duas estações do ano. Em relação à massa total, nota-se que há constante queda de folhas, galhos, flores e frutos durante todo o ano, sendo a fração foliar (73,6\% do total de liteira) a mais representativa, seguida de galhos $(25,0 \%)$, frutos $(3,9 \%)$ e flores $(3,9 \%)$.

Através da análise de Fisher's LSD, a queda de folhas foi significativamente maior nos períodos chuvososeco e seco $(p<0,001)$, e os pares não diferiram significativamente $(p>0,05)$ entre si (Figura2). Não foi observada influência dos níveis topográficos $(p>0,05)$. A queda de flores foi significativamente menor no platô durante o período seco-chuvoso ( $p$ $<0,01)$, sem variação significativa $(\mathrm{p}>0,05)$ entre o nível de baixio e o intermediário nem entre os períodos seco-chuvoso, chuvoso e seco. Apenas nos períodos seco e seco-chuvoso houve diferença significativa $(\mathrm{p}<0,05)$ na queda de flores entre os níveis (Figura 2). Foi encontrada diferença significativa na queda de frutos $(\mathrm{p}<0,001)$ entre baixio e intermediário, durante os períodos seco-chuvoso e chuvoso e seco. No período chuvoso, a produção de frutos no baixio foi significativamente maior $(\mathrm{p}<0,01)$. No período chuvososeco, o nível intermediário diferiu significativamente $(\mathrm{p}<0,05)$ quando comparado ao platô (Figura2). Os resultados revelaram que a produção de frutos na Flona de Caxiuanã tem preferência para ocorrer entre o período seco-chuvoso e chuvoso em todos os níveis topográficos. A queda de galhos no baixio diferiu significativamente $(p<0,01)$ (Figura 2$)$. Em relação aos períodos sazonais, no período seco-chuvoso a

Revista Árvore, Viçosa-MG, v.39, n.6, p.995-1005, 2015 
Tabela 1 - Espécies com Índice de Valor de Importância (IVI) nos níveis topográficos de baixio, intermediário e platô na FLONA de Caxiuanã, Pará.

Table 1 - Species with Importance Value Index (IVI) in topographic levels of Lowland, Intermediate and Plateau in FLONA Caxiuanã, Melgaço, Pará.

\begin{tabular}{|c|c|c|c|}
\hline \multirow{2}{*}{ Espécies } & \multicolumn{3}{|c|}{ Nível topográfico } \\
\hline & Baixio & Intermediário & Platô \\
\hline Abarema mataybifolia (Sandwith) Barneby \& J.W. Grimes & & 0,69 & \\
\hline Amajoua sp. & 0,68 & & \\
\hline Aniba kappleri Mez & & 0,95 & 0,96 \\
\hline Aniba parviflora (Meisn.) Mez & 0,67 & 0,62 & \\
\hline Astronium gracile Engl. & & 0,62 & \\
\hline Astronium lecointei Ducke & & 2,45 & \\
\hline Balizia pedicellaris (DC.) Barneby \& J.W. Grimes & 2,58 & & \\
\hline Bocageopsis multiflora (Mart.) R.E. Fr. & & 0,64 & 0,76 \\
\hline Brosimum acutifolium Huber & & & 1,34 \\
\hline Brosimum guianense Huber ex Ducke & & 0,85 & \\
\hline Brosimum parinarioides Ducke & & & 0,89 \\
\hline Caryocar glabrum Pers. & & & 1,46 \\
\hline Chaunochiton kappleri (Sagot ex Engl.) Ducke & & 1,41 & \\
\hline Chimarrhis turbinata DC. & & & 3,30 \\
\hline Chrysophyllum prieurii A. DC. & 2,20 & & 1,38 \\
\hline Clarisia racemosa Ruiz \& Pav. & 1,52 & & 0,95 \\
\hline Copaifera reticulata Ducke & & 1,00 & 0,82 \\
\hline Couratari stellata A.C. Sm. & & & 5,80 \\
\hline Cupania hirsuta Radlk. & & 0,62 & \\
\hline Dialium guianense Steud. & & 0,79 & \\
\hline Dinizia excelsa Ducke & & & 0,84 \\
\hline Diospyros mellinonii (Hiern) A.C. Sm. & 0,70 & & \\
\hline Dipterix sp. & 0,99 & & \\
\hline Dodecastigma integrifolium (Lanj.) Lanj. \& Sandwith & 1,03 & 1,43 & \\
\hline Duguetia echinophora R.E. Fr. & & & 0,72 \\
\hline Ecclinusa ramiflora Mart. & 1,09 & 0,62 & \\
\hline Endopleura uchi (Huber) Cuatrec. & & 1,71 & \\
\hline Eschweilera albiflora (DC.) Miers & & & 0,73 \\
\hline Eschweilera amazonica $\mathrm{R}$. Kunth & 3,53 & 1,47 & 2,05 \\
\hline Eschweilera coriacea (DC.) S.A. Mori & 3,46 & 6,10 & 5,52 \\
\hline Eschweilera grandifolia Mart. ex Doc. & 1,38 & & \\
\hline Eschweilera micrantha (O. Berg) Miers & & 0,63 & 0,78 \\
\hline Eschweilera pedicellata (Rich.) S.A. Mori & & & 0,76 \\
\hline Eugenia flavescens Ridl. & & 0,61 & \\
\hline Eugenia patrisii Vahl & 0,71 & 0,70 & \\
\hline Geissospermum sericeum Miers & & 1,07 & 1,73 \\
\hline Goupia glabra Aubl. & 2,40 & & \\
\hline Guarea kunthiana A. Juss. & & 0,65 & \\
\hline Guatteria poeppigiana Mart. & & 0,94 & 0,73 \\
\hline Hebepetalum humirifolium (Planch.) Benth. & & & 0,72 \\
\hline Hevea brasiliensis (Willd. ex A. Juss.) Müll. Arg. & & & 1,41 \\
\hline Hirtella bicornis Mart. \& Zucc. & & 0,62 & 0,73 \\
\hline Hymenaea courbaril L. & 1,42 & & \\
\hline Hymenolobium flavum Kleinhoonte & 0,66 & & \\
\hline Iryanthera laevis Markgr. & & & 0,76 \\
\hline Iryanthera paraensis Huber & 0,90 & & \\
\hline Iryanthera sagotiana (Benth.) Warb. & & 0,76 & \\
\hline
\end{tabular}


Tabela 1 - Cont

Table 1 - Cont

\begin{tabular}{|c|c|c|c|}
\hline \multirow{2}{*}{ Espécies } & \multicolumn{3}{|c|}{ Nível topográfico } \\
\hline & Baixio & Intermediário & Platô \\
\hline Jacaranda copaia (Aubl.) D. Don & & 0,69 & \\
\hline Lecythis idatimon Aubl. & 7,58 & 4,85 & 5,45 \\
\hline Lecythis jarana (Huber ex Ducke) A.C. Sm. & & 2,76 & \\
\hline Lecythis lurida (Miers) S.A. Mori & 0,93 & 0,73 & \\
\hline Licania apetala (E. Mey.) Fritsch & 1,78 & 1,92 & \\
\hline Licania canescens Benoist & 1,42 & 1,26 & 1,46 \\
\hline Licania egleri Prance & 1,38 & 1,34 & 1,81 \\
\hline Licania gracilis Kleinh. & & 1,93 & \\
\hline Licania heteromorpha Benth. & 0,67 & 0,65 & 1,69 \\
\hline Licania membranacea Sagot ex Laness. & 2,88 & 0,78 & 1,81 \\
\hline Licania octandra (Hoffmanns. ex Roem. \& Schult.) Kuntze & 1,80 & 2,54 & \\
\hline Mabea angularis Hollander & & 1,22 & \\
\hline Mabea caudata Pax \& K. Hoffm. & 1,42 & 0,73 & \\
\hline Macrolobium microcalyx Ducke & 2,17 & 3,33 & 0,73 \\
\hline Manilkara bidentata (A. DC.) A. Chev. & 2,03 & 3,06 & 2,76 \\
\hline Manilkara huberi (Ducke) Standl. & 1,42 & & \\
\hline Maprounea guianensis Aubl. & & 0,78 & \\
\hline Mezilaurus itauba (Meisn.) Taub. ex Mez & & & 0,81 \\
\hline Micropholis acutangula (Ducke) Eyma & & 1,97 & 2,82 \\
\hline Micropholis guyanensis (A. DC.) Pierre & 0,70 & 0,63 & 1,28 \\
\hline Micropholis venulosa (Mart. \& Eichler) Pierre & & 0,90 & 1,30 \\
\hline Minquartia guianensis Aubl. & 1,91 & 0,92 & 1,10 \\
\hline Myrcia atramentifera Barb. Rodr. & & 1,39 & \\
\hline Naucleopsis krukovii (Stand1.) C.C. Berg & 1,20 & & 0,75 \\
\hline Ocotea canaliculata (Rich.) Mez & & 0,63 & 0,72 \\
\hline Ocotea glomerata (Nees) Mez & & 0,83 & 2,29 \\
\hline Ocotea parviflora (Ducke) Kosterm & & & 0,94 \\
\hline Osteophloeum platyspermum (Spruce ex A. DC.) Warb. & 2,40 & & 0,77 \\
\hline Parinari excelsa Sabine & 2,13 & 0,85 & 1,41 \\
\hline Poecilanthe effusa (Huber) Ducke & & 0,86 & 1,80 \\
\hline Pourouma guianensis Aubl. & 2,28 & & \\
\hline Pourouma villosa Trécu & & 1,09 & \\
\hline Pouteria anomala (Pires) T.D. Penn. & 2,20 & 4,52 & 1,07 \\
\hline Pouteria cladantha Sandwith & 4,23 & 3,23 & 3,11 \\
\hline Pouteria decorticans T.D. Penn. & 0,68 & & \\
\hline Pouteria eugeniifolia (Pierre) Baehni & & & 0,84 \\
\hline Pouteria filipes Eyma & 1,18 & & \\
\hline Pouteria gongrijpii Eyma & 2,66 & 1,07 & 1,43 \\
\hline Pouteria guianensis Aubl. & 1,25 & 0,62 & 0,75 \\
\hline Pouteria krukovii (A.C. Sm.) Baehni & 0,87 & & 0,93 \\
\hline Pouteria oppositifolia (Duck) Baehni & 1,84 & & \\
\hline Pouteria retinervis T.D. Penn. & 0,77 & 0,67 & \\
\hline Protium decandrum (Aubl.) Marchand & 0,86 & & \\
\hline Protium paniculatum Engl. & & 0,89 & \\
\hline Protium robustum (Swart) D.M. Porter & & & 0,71 \\
\hline Protium spruceanum (Benth.) Engl. & 0,66 & & \\
\hline Pseudolmedia laevigata Trécul & & 0,65 & \\
\hline Pseudopiptadenia suaveolens (Miq.) J.W. Grimes & & 1,88 & \\
\hline Quiina florida Tul. & & & 1,21 \\
\hline
\end{tabular}


Tabela 1 - Cont.

Table 1 - Cont.

\begin{tabular}{|c|c|c|c|}
\hline \multirow{2}{*}{ Espécies } & \multicolumn{3}{|c|}{ Nível topográfico } \\
\hline & Baixio & Intermediário & Platô \\
\hline Rinorea guianensis Aubl. & 4,22 & 6,17 & 3,33 \\
\hline Rinorea apiculata Hekking & & 0,62 & \\
\hline Roupala montana Aubl. & 1,02 & & \\
\hline Saccoglotis guianensis & 0,68 & 0,89 & 2,13 \\
\hline Sagotia racemosa Baill. & & & 0,72 \\
\hline Simaruba sp. & & 1,05 & \\
\hline Sloanea eichleri $\mathrm{K}$. Schum. & & 0,62 & \\
\hline Sloanea guianensis (Aub1.) Benth. & & 1,22 & \\
\hline Sloanea porphyrocarpa Ducke & 0,76 & & 1,14 \\
\hline Sloanea parviflora Planch. ex Benth. & 0,67 & & \\
\hline Stryphnodendron paniculatum Poepp. & & 1,20 & 0,94 \\
\hline Swartzia grandifolia Bong. ex Benth. & & 0,61 & \\
\hline Symphonia globulifera L. f. & & & 0,76 \\
\hline Tachigali myrmecophila (Ducke) Ducke & 0,67 & & \\
\hline Tachigali paraensis (Huber) Barneby & 0,72 & & \\
\hline Tetragastris altissima (Aubl.) Swart & 0,71 & 0,83 & 2,48 \\
\hline Tetragastris panamensis (Engl.) Kuntze & 2,74 & 0,61 & 2,88 \\
\hline Theobroma subincanum Mart. & & & 1,13 \\
\hline Trattinnickia rhoifolia Willd. & 0,70 & & \\
\hline Trichilia micrantha Benth. & & 0,62 & \\
\hline Vantanea parviflora Lam. & 0,85 & 0,77 & \\
\hline Vatairea erythrocarpa (Ducke) Ducke & 0,67 & & \\
\hline Vouacapoua americana Aubl. & 4,92 & 4,02 & 5,91 \\
\hline Xylopia emarginata Mart. & 0,66 & & \\
\hline Zygia racemosa (Ducke) Barneby \& J.W. Grimes & & 0,71 & 1,70 \\
\hline
\end{tabular}

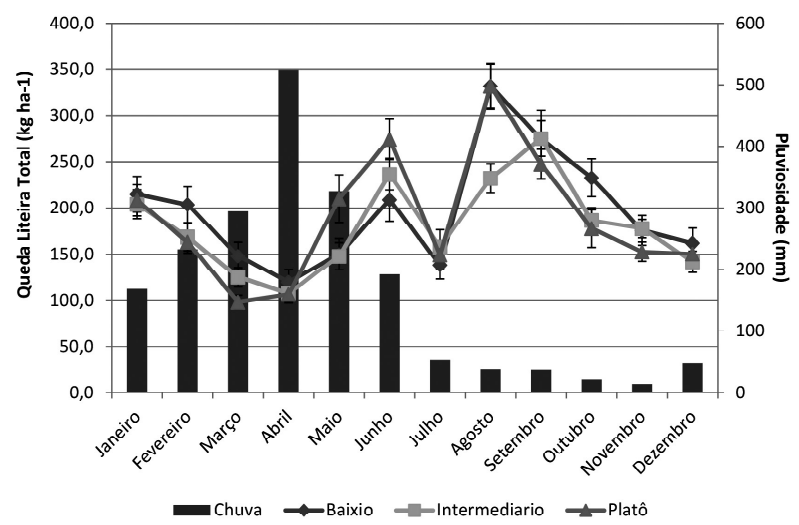

Figura 1 - Variação mensal da produção de liteira total e pluviosidade nos níveis topográficos de Baixio, Intermediário e Platô na FLONA de Caxiuanã, Melgaço, Pará.

Figure 1-Monthly variation of total litter production and rainfall in topographic levels of lowland, Intermediate and Plateau in FLONA Caxiuanã, Melgaço, Pará.

Revista Árvore, Viçosa-MG, v.39, n.6, p.995-1005, 2015 queda de galhos foi significativamente maior $(\mathrm{p}<0,01)$ do que nos outros períodos analisados, e o período seco e chuvoso não diferiu entre si, os quais foram significativamente diferentes $(\mathrm{p}<0,01)$ do período chuvoso-seco, quando foi encontrada a menor queda.

\section{DISCUSSÃO}

\subsection{Composição Florística}

Em um estudo de comparação estrutural e florística em dois tipos de ambientes (terra-firme e várzea) em Caxiuanã, foi constatado que a floresta de terra firme ocupa cerca de $85 \%$ da área (VIANA et al., 2003). Muito embora os autores não tenham dividido em diferentes topografias, constataram que a diversidade variou entre 150 e 160 espécies ha ${ }^{-1}$, a densidade de 450 a 550 árvores $\mathrm{ha}^{-1}$ e a biomassa acima do solo com $200 \mathrm{~m}^{3} \mathrm{ha}^{-1}$, resultados muito acima daqueles registrados nesta pesquisa. Apenas Eschweilera coriacea (Ap. Dec.) Martius ex Berg (Lecythidaceae), Voucapoua americana 


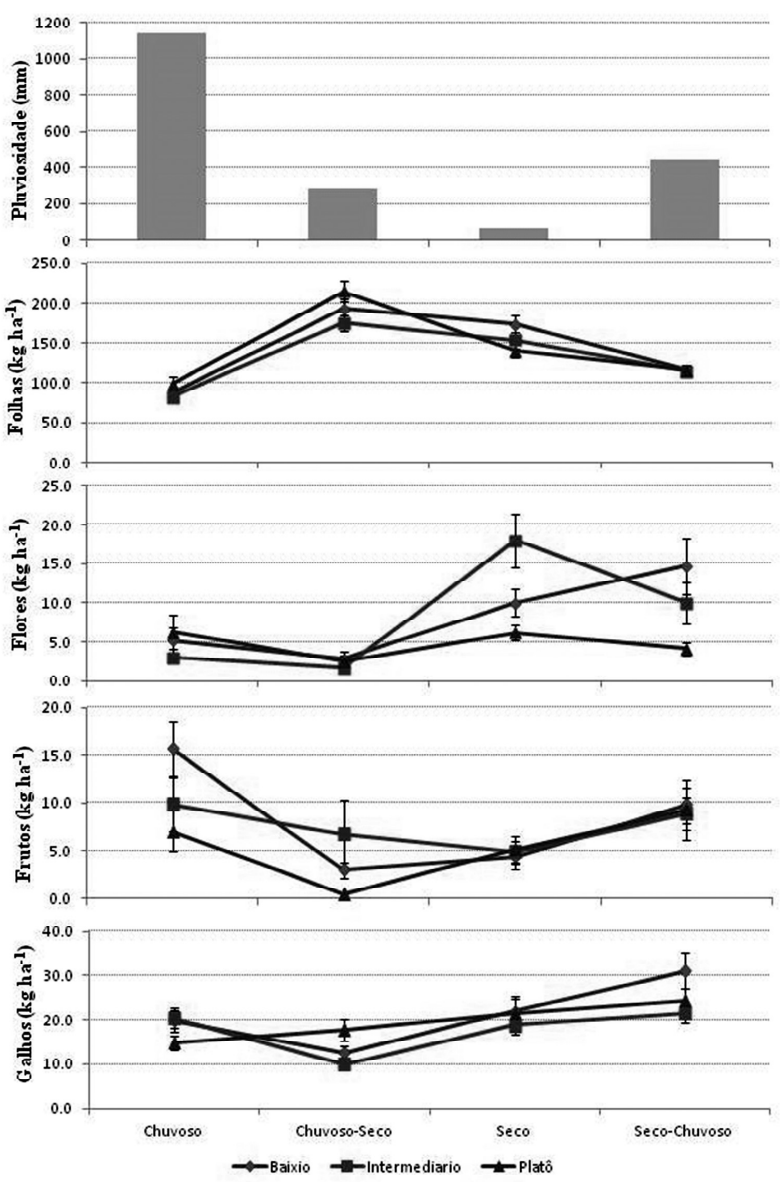

Figura 2 - Variação sazonal da pluviosidade em relação à queda de folhas, flores, frutos e galhos nos níveis topográficos de Baixio, Intermediário e Platô na FLONA de Caxiuanã, Melgaço, Pará.

Figure 2-Seasonal rainfall variation in relation to leaf fall, flowers, fruits and branches in topographic levels of Lowland, Intermediate and Plateau in FLONA Caxiuanã, Melgaço, Pará.

Aublet (Caesalpiniaceae) e Protium pallidum Cuatrec (Burseraceae) corroboraram com as espécies deste trabalho. Estudos de Amaral et al. (2009) na floresta de terra firme de Caxiuanã evidenciaram uma riqueza entre 150 e 180 espécies de árvores ha ${ }^{-1}$, com área basal média de $31,2 \mathrm{~m}^{2} \mathrm{ha}^{-1} \mathrm{e}$ volume médio de madeira ao redor de $300 \mathrm{~m}^{3} \mathrm{ha}^{-1}$, cujas espécies com maior índice de valor de importância: Eschweilera coriacea (Lecytidaceae), Rinorea guianensis (Violaceae), Eschweilera collina (Lecytidaceae), Lecythis idatimon (Lecythidaceae) e Vouacapoua americana (Caesalpiniaceae) também foram registradas neste estudo.
Para Souza et al. (2012), a distribuição das espécies ao longo do gradiente topográfico está associada com as variações da fertilidade química, acidez e textura do solo.

\subsection{Produção de Liteira}

Diferentemente deste trabalho, Borem e Ramos (2002), estudando a liteira em fragmentos de floresta de Mata Atlântica, registraram maior estoque de liteira nos terços médio e superior de uma topossequência de floresta devido ao maior conteúdo de nutrientes e de carbono orgânico apresentado por essas posições topográficas. Outra relação que os autores observaram foi que o terço inferior da topossequência pouco alterada apresentou valores de acumulação de liteira mais elevados que os terços médio e superior. A variação mensal de liteira em floresta natural na FLONA de Caxiuanã encontrada por Silva et al. (2009) foi de $0,8 \mathrm{~kg} \mathrm{ha}^{-1}$, valor aproximado ao deste estudo. Na Floresta Nacional de Tapajós, Brando et al. (2008), estudando uma floresta primária, constataram maior produção de liteira no período de menor precipitação. A mesma sazonalidade na produção da liteira, descrita neste trabalho, foi encontrada por Silva et al. (2009), e nas duas pesquisas feitas na mesma área (Flona de Caxiuanã) mostrou maior produção de liteira entre as estações chuvosas e menos chuvosa, discordando, parcialmente, dos resultados apresentados por Malhi et al. (2009). A influência sazonal sobre a queda de liteira é esperada para os ecossistemas amazônicos (LUIZÃO, 1989; MALHI et al., 1998; HUETE et al., 2006; CHAVE et al., 2010). Segundo Silva et al. (2009), essa maior quantidade de queda de liteira no período seco pode ser resultado do aumento da radiação solar durante esse período, o que pode aumentar a produtividade primária em florestas tropicais, ou seja, as plantas mantêm suas folhas novas, descartando as folhas velhas, para maior eficiência na produtividade primária líquida.

A relação inversa entre disponibilidade hídrica, menor no período seco e a grande área foliar em floresta tropical, reflete a importante estratégia de minimizar os efeitos da escassez de água, por meio de intensificação da queda de liteira na floresta e produção de folhas novas com mais agilidade para controle das perdas de água. Segundo Huete et al. (2006), essa perda de grande quantidade de folhas durante o período seco pode ser resultado do estresse hídrico sofrido pela planta, que perde folhas para minimizar o efeito da

Revista Árvore, Viçosa-MG, v.39, n.6, p.995-1005, 2015 
escassez de água, porém a grande queda das folhas é motivada pela sazonalidade da radiação solar, em vez das chuvas (WRIGHT; VAN SHAIK, 1994). Os dados obtidos nesta pesquisa revelam que não parece ser o estresse hídrico do solo o fator a acionar o gatilho para a queda das folhas e, sim, o fato de que, com a chegada do período seco, há uma necessidade de se aumentar o controle de perda de água pelos estômatos, devido à diminuição da umidade relativa do ar e, com isso, aumentar a eficiência fotossintética

Fatores abióticos são fortemente correlacionados com o florescimento de espécies e, em alguns casos, até mesmo a fisiologia dos indivíduos pode influenciar essa floração. Nas regiões neotropicais sazonais, as espécies arbóreas apresentam flores tanto na estação seca quanto na estação chuvosa, e algumas espécies não produzem flores no período de um ano (RATHCKE; LACEY, 1985). Em florestas tropicais em que as árvores florescem em resposta à precipitação, as chuvas podem causar variação desigual no hábitat, provocando assincronia no florescimento entre espécies e dentro das espécies (AUGSPURGER, 1983). A floração em massa em florestas tropicais é comum entre árvores que florescem durante a estiagem, enquanto a floração de estado estacionário é encontrada na maioria das espécies de sub-bosque (JANZEN, 1967; GENTRY, 1974; FRANKIE et al., 1974; AUGSPURGER, 1983; BAWA, 1983).

Ao contrário de Borchert (1983), os resultados revelaram que há preferência para iniciar o processo reprodutivo ao final do período chuvoso-seco, intensificando-se no período seco e atingindo o período seco-chuvoso, principalmente nas topografias de baixio e intermediária. Entretanto, esse padrão não está bem claro na topografia de platô, ou seja, existe produção constante de flores para essa topografia, independentemente da estação do ano. Para Tonello et al. (2014), a distribuição da chuva mostra diferenças significativas nos povoamentos florestais. Assim, é importante ressaltar que a diferença na estrutura da população, quando comparado o baixio com o platô, revela estratégias distintas para a produção de flores ou, mais especificamente, que a diversidade de indivíduos dentro das espécies pode estar sendo maior nas populações que vivem nas áreas de platô, resultando em produção diferenciada de flores dentro da população.

Em florestas tropicais sazonais, onde a permanência das sementes no solo deve ser o mínimo possível
(GARWOOD, 1983), algumas espécies de árvores amadurecem e liberam seus frutos no fim da estação seca, quando os ventos alísios são fortes e muitas folhas caem (JANZEN, 1967; CROAT, 1969; SMYTHE, 1970; LIEBERMAN, 1982). Essa estratégia pode minimizar o tempo que as sementes permanecem no solo, para poder germinar no início da estação chuvosa, o que condiz com outros trabalhos realizados na Amazônia, os quais afirmam que as espécies com dispersão zoocóricas apresentam um pico de maturação dos frutos durante a estação chuvosa (JANZEN, 1967; KARR, 1976).

\section{CONCLUSÃO}

A influência da sazonalidade climática, principalmente na transição entre os dois períodos sazonais, interferiu intensamente na produção de liteira, revelando a maior produção no final da estação chuvosa e no início da estação seca. A menor disponibilidade hídrica no solo reflete importante estratégia para minimizar os efeitos da escassez de água no ambiente, por meio de intensificação na produção de folhas novas com mais agilidade, para controle das perdas de água para a atmosfera. Assim, a queda foliar é decorrente, sobretudo, da diminuição da umidade relativa do ar, que dificulta a eficiência fotossintética. A diferença na estrutura da população, quando comparado, principalmente, o baixio com o platô, revelou estratégias distintas para a produção de flores ou, mais especificamente, que a diversidade de indivíduos dentro das espécies pode estar sendo maior nas populações que vivem nas áreas de platô, resultando em produção diferenciada de flores dentro da população.

\section{REFERÊNCIAS}

ALMEIDA, S.S.; LISBOA, P.L.B.; SILVA, A.S.L. Diversidade florística de uma comunidade arbórea na Estação Científica Ferreira Penna, em Caxiuanã (Pará). Boletim do Museu Paraense Emílio Goeldi, série Botânica, v.9, n.1, p.99-188, 1993.

AMARAL, D.D.; ALMEIDA, S.S.; COSTA, D.C.T. Contribuições ao manejo florestal de espécies de valor madeireiro e não madeireiro na Floresta Nacional de Caxiuanã. In: LISBOA, P.L.B. (Org.) Caxiuanã: Desafios para a conservação de uma Floresta Nacional na Amazônia. Belém: Museu Paraense Emílio Goeldi, 2009. p.199-221. 
ARAGÃO, L.E.O.C.; MALHI, Y.; METCLAFE, D.B. Above and below-ground net primary productivity across ten Amazonian Forests on contrasting soils. Biogeosciences

Discussions, v.6, n.2, p.2441-2488, 2009.

ASNER, G.P.; TOWNSEND, A.R.; BRASWELL, B.H. Satellite observation of El Niño effects on Amazon forest phenology and productivity. Geophysical Ressearch Letters, v.27, n.1, p.981-984, 2000.

AUGSPURGER, C.K. Phenology, flowering synchrony, and fruit set of six neotropical shrubs. Biotropica, v. 15, n.4, p.257-267, 1983.

BAWA, K. S. Patterns of flowering in tropical plants. In: JONES, C.E.; LITTLE, R.J. (Eds.).

Handbook of experimental pollination biology. New York: Scientific and Academic, 1983. p.394-410.

BORCHERT, R. Phenology and control of flowering in tropical trees. Biotropica, v.5, n.2, p.81-89, 1983 .

BORÉM, R.A.T.; RAMOS, D.P. Variação estacional e topográfica de nutrientes na serrapilheira de um fragmento de Mata Atlântica. Cerne, v.8, n.2, p.42-59, 2002.

BRANDO,P.M.; NEPSTAD, D.C.; DAVIDSON, E.A.; TRUMBORE, S.E.;RAY, D.; CAMARGO, P. Drought effects on litterfall, wood production and belowground carbon cycling in a Amazon forest: results of a throughfall reduction experiment. Biological Sciences, v.363, p.1839-1848, 2008

BURGHOUTS, T.B.A.; CAMPBELL, E.J.F.; KODERMAN, P.J. Effects of tree species heterogeneity of leaf fall in primary and logged dipterocarp forest in the UluSegana Forest Reserve, Sabah, Malasia. Journal of Tropical Ecology, v.10, p.1-26, 1994.

CHAVE, J.; NAVARRETE, D.; ALMEIDA,S.; ÁlVAREZ, E.; ARAGÃO, L.E.O.C.; BONAL, D.; CHÂTELET, P.; SILVA-ESPEJO, J.E.; GORET, J.Y.; von HILDEBRAND, P.; JIMÉNEZ, E.; PATIÑO, S.; PEÑUELA, M.C.; PHILLIPS, O.L.;
STEVENSON, P.; MALHI, Y. Regional and seasonal patterns of litterfall in tropical South America. Biogeosciences, v.7, p.43-55, 2010.

COSTA, A.C.L.; ALMEIDA, S.S.; CARVALHO, C.R.; MEIR, P.; MALHI, Y.; BRAGA, A.P.; BARRETO, P.N. Experimento Esecaflor-LBA em Caxiuanã. In: LISBOA, P.L.B. (Org.)

Caxiuanã: desafios para a conservação de uma Floresta Nacional na Amazônia. Belém: Museu Paraense Emílio Goeldi, 2009. p. 45-90.

CROAT, T. B. Seasonal flowering behavior in central Panama. Annual Monografe

Botanical Garden, v.56, p.295-307, 1969.

DIAS, H.C.T.; OLIVEIRA FILHO, A.T. Variação temporal e espacial da produção de liteira em uma área de Floresta Estacional Semidecídua Montana em Lavras-MG. Revista Árvore, v.21, n.1, p.11-26, 1997.

FRANKIE, G.W.; BAKER, H.G.; OPLER, P.A. A comparative phenological studies of trees in tropical wet and dry forest in the lowlands of Costa Rica. Journal of Ecology, v.62, n.3, p.881-919, 1974.

GARWOOD, N.C. Seed germination in a seasonal tropical forest in Panama: A community study. Ecology Monograph, v.53, p.159$181,1983$.

GENTRY, H.A. Flowering phenology and diversity in tropical Bignoniaceae.

Biotropica, v.6, p.64-68, 1974.

HUETE, A.R.; DIDAN, K.; SHIMABUKUKO, Y.E; RATANA, P.; SULESKA, S.R.; HUTYRA, L.R.; YANG, W.Z.; NEMANI, R.R.; MYNENI,R. Amazon rainforest gree-up with Sun-light in dry season. Geophysical Research Letters, v.33, p.1-4, 2006.

JANZEN, D.H. Synchronization of sexual reproduction of trees within the dry season in Central America. Evolution, v.21, n.3, p.620637, 1967.

KARR, J.R. Seasonality, resource availability, and community diversity in tropical bird communities. The American Naturalist, v.110, p.973-994, 1976.

Revista Árvore, Viçosa-MG, v.39, n.6, p.995-1005, 2015 
LIEBERMAN, D. Seasonality and phenology in a dry forest in Ghana. Journal Ecology, v.70, p.791-806, 1982.

LUIZÃO, F.J. Litter production and mineral element input to the forest floor in a central Amazonian forest. Geological Journal, v.19, p.407-417, 1989.

MALHI, Y.; ARAGÃO, L.E.O.C.; METCALFE, D.B.; PAIVA, R.; QUESADA,C.A.; ALMEIDA, S.; ANDERSON, L.; BRANDO, P.; CHAMBERS, J.Q.; DA COSTA, A.C.L.; HUTYRA, L.R.; OLIVEIRA, P.; PATIÑO, S.; RYLE, E.H.; ROBERTSON, A.L.; TEIXEIRA, L.M. Comprehensive assessment of carbon productivity, allocation and storage in three Amazonian forests. Global Change Biology, v.15, n.1, p.1255-1274, 2009.

MALHI, Y.; NOBRE, A.D.; GRACE, J.; KRUIJT, B.; PEREIRA, M.G.P.; CUEF, A.; SCOTT, S.

Carbondioxidetransfer over a Central Amazonianrain Forest. Journal of Geophysical Research, v.103, p.31593-31612, 1998.

MATTEUCCI, S.D.; COLMA, A.; MIRANDA, F. Metodologia para el estudio de la vegetacion. Program Regional de Desarrollo Científicos y Tecnológico Washington, D.C.: 1982. (Monografias, Série Biológica, 22).

METCALFE, D.; MEIR, P.; ARAGÃO, L.E.O.C.; DA COSTA, A.; ALMEIDA, S.; BRAGA, A.; GONÇALVES, P.; ATHAYDES, J.; MALHI, Y.; WILLIAMS, M. Sample sizes for estimating key ecosystem characteristics in a tropical terra firme rainforest. Forest Ecology and

Management, v.255, p.558-566, 2008.

NOVAIS, R.F.; BARROS, N.F. Sustainable agriculture and forestry production systems on acid soils: phosphorus as a case study. In: MONIZ, A.C. (Ed.) Plant-soil interactions at low Ph: Sustainable agriculture and forestry productions. Campinas: Brazilian Soil Science Society, 1997. p. 314-320.

PEDRONI, F.; SANCHEZ, M.; SANTOS, F.A.M. Fenologia da Copaíba (Copaifera langsdorffii Desf. - Leguminosae, Caesalpinioideae) em uma floresta semidecídua no sudeste do Brasil.

Revista Árvore, Viçosa-MG, v.39, n.6, p.995-1005, 2015
Revista Brasileira de Botânica, v.25, n.2, p.183-194, 2002.

PEEL, M.C.; FINHAYSON, B.L.; McMANHON, T.A. Updated world map of the Köppen-Geiger climate classification. Hydrology and Earth System Sciences, v.11, p.1633-1644, 2007.

PORTES, M.C.G.O.; KOEHLER, A.; GALVÃO, F. Variação sazonal de deposição de serapilheira em uma Floresta Ombrófila Densa Altomontana no morro do Anhagava- PR. Floresta, v.26, n.1, p.3$10,1996$.

SMYTHE, N. Relationships between fruiting seasons and seed dispersal methods in a neotropical forest. The American Naturalist, v.104, p.25-35, 1970.

RATHCKE, B.; LACEY, E.P. Phenological patterns of terrestrial plants. Annual Review of Ecology and Systematics, v.16, p.179-214, 1985.

REICH, P.B. Phenology of tropical forest patterns, causes, and consequences. Canadian Journal of Botany, v.73, p.164-174, 1995.

SANCHEZ-AZOFEIFA, A.; KALACSKA, M.; QUESADA, M.; STONER, K.; LOBO, J.; ARROYOMORA, P. Tropical dry climates. In: SCHWARTZ, M.D. (Ed.). Phenology: an intergration environmental science. London: Kluwer, 2003. p.121-138.

SILVA, C.J.; LOBO, F.A.; BLEICH, M.E.; SANCHES, L. Contribuição de folhas na formação de serrapilheira e no retorno de nutrientes em floresta de transição no norte de Mato Grosso. Acta Amazonica, v.39, n.3, p.591-600, 2009.

SOUZA,P.B.; LELIS, J.J.; SCHAEFER, C.E.G.R.; SOUZA, A.L.; MEIRA-NETO, J.A.A. Distribution of tree species in a geomorphological and pedological gradient of submontane semidecidual seasonal forest in the vicinity of Rio Doce State Park, Minas Gerais. Revista Árvore, v.36, n.4, p.707-718, 2012.

TONELLO, K.C.; GASPAROTO, E.A.G.; SHINZATO; VALENTE, R.O.A.; DIAS, H.C.T. Precipitação efetiva em diferentes formações florestais na Floresta Nacional de Ipanema. Revista Árvore, v.38, n.2, p.383-390, 2014. 
VIANA, J.S.; ALMEIDA,S.S.; CONCEIÇÃO,C.; FERREIRA,E.; ALVES,N.E.; SILVA, R. Comparação estrutural e florística entre os ambientes de terrafirme e igapó do rentorno da Estação Científica Ferreira Penna - ECFPn. In: MONTAG, L.F.A.; MASCHIO, G.F. (Eds.). Dez anos de pesquisa na Amazônia, Sessão I: Dez anos de pesquisa na Estação Científica Ferreira Penna, Caxiuanã. Belém: MPEG, 2003.

VITOUSEK, P.M.; SANFORD Jr., R.L. Nutrient cycling in moist tropical forest. Annual
Review of Ecology and Systematics, v.17, n.1, p.137-167, 1986.

WERNECK, M.S.; PEDRALLI, G.; GIESEKE, L.F. Produção de serrapilheira em trechos de uma floresta semidecídua com diferentes graus de perturbação na estação Ecológica do Tripuí, Ouro Preto-MG. Revista Brasileira de Botânica, v.24, n.2, p.195-198, 2001.

WRIGHT, S.J.; van SCHAIK, C.P. Light and the phenology of tropical trees. American Naturalist, v.143, p.192-199, 1994. 
